\title{
ADHERENCIA TERAPÉUTICA EN USUARIOS DE UN PROGRAMA DE SALUD CARDIOVASCULAR DE ATENCIÓN PRIMARIA EN CHILE
}

\author{
Lizet Veliz-Rojas ${ }^{1, a}$, Sara Mendoza-Parra ${ }^{2, b}$, Omar A. Barriga ${ }^{3, c}$
}

RESUMEN

Objetivos. Analizar la adherencia terapéutica en usuarios de un programa de salud cardiovascular de atención primaria de la comuna de San Pedro de la Paz en la región del Bío Bío, Chile. Materiales y métodos. Estudio de transversal y correlacional, con una muestra de 257 personas de 18 a 60 años. Se utilizó un cuestionario que incluía la escala de conductas en salud de Miller para medir adherencia terapéutica, y la revisión de la ficha clínica. Se utilizó un análisis descriptivo univariante y bivariado apoyado en SPSS. Resultados. Del total de participantes $157(61,1 \%)$ fueron mujeres. La Escala de conductas en salud reflejó la no adherencia de los participantes, ya que solo 4 (1,5\%) personas indicaron que siempre seguían las indicaciones entregadas por el equipo de salud. La subescala seguimiento en el manejo del estrés tuvo la media más alta, lo que indica que en este aspecto existió una mayor adherencia en los participantes. Se encontraron asociaciones entre la adherencia terapéutica con la realización de un trabajo remunerado $(p=0,025)$ y con la participación de actividades sociales $(p=0,005)$.Conclusiones. La adherencia terapéutica en los usuarios del programa de salud cardiovascular fue baja. Es importante desarrollar estrategias que favorezcan la adherencia terapéutica desde la perspectiva de equidad y de los determinantes sociales de la salud.

Palabras clave: Cooperación del paciente; Enfermedades cardiovasculares; Atención primaria de salud (fuente: DeCS BIREME).

\section{THERAPEUTIC ADHERENCE IN USERS OF A CARDIOVASCULAR HEALTH PROGRAM IN PRIMARY CARE IN CHILE}

\begin{abstract}
Objectives. To analyze therapeutic adherence in users of a cardiovascular health program in primary care in the community of San Pedro de la Paz in the region of Bío Bío, Chile. Materials and methods. Cross-sectional and correlational study with a sample of 257 people aged 18-60 years. A questionnaire that included the Miller's health behavior scale to measure adherence, and review of medical records was performed. Descriptive univariate and bivariate analyses supported in SPSS were performed. Results. Of the total participants, 157 (61.1\%) were women. The health behavior scale reflected non-adherence of participants, as only $4(1.5 \%)$ indicated that they always followed the instructions provided by the health team. The subscale monitoring stress management had the highest average, indicating that in this aspect there was greater adherence of the participants. Associations between therapeutic adherence and doing paid work $(p=0.025)$ and with participation in social activities $(p=0.005)$ were found. Conclusions. Therapeutic adherence in users of the cardiovascular health program was low. It is important to develop strategies that favor therapeutic adherence from the perspective of equity and social determinants of health.
\end{abstract}

Key words: Patient compliance; Cardiovascular diseases; Primary health care (source: MeSH NLM).

\footnotetext{
Facultad de Medicina, Universidad Católica del Norte. Coquimbo, Chile.

Departamento de Enfermería, Universidad de Concepción. Chile.

Departamento de Sociología y Antropología, Universidad de Concepción. Chile.

a Enfermera, doctora en Enfermería, magíster en Salud Pública; ${ }^{\mathrm{b}}$ enfermera, doctora en Enfermería; ${ }^{\mathrm{c}} \mathrm{PhD}$ en Sociología

Este estudio formo parte de la tesis titulada "Automedicación y adherencia terapéutica como determinantes del control integral de las enfermedades cardiovasculares" para obtener el grado de doctor en Enfermería.

Recibido: 21-06-14 Aprobado: 11-02-15
}

Citar como: Veliz-Rojas L, Mendoza-Parra S, Barriga OA. Adherencia terapéutica en usuarios de un programa de salud cardiovascular de atención primaria en Chile. Rev Peru Med Exp Salud Publica. 2015;32(1):51-7 


\section{INTRODUCCIÓN}

En las últimas décadas, la adherencia terapéutica ha adquirido un papel relevante en la literatura científica, aunque ya desde hace siglos era un problema señalado. Son conocidos los comentarios de Platón sobre la importancia de incidir sobre la correcta toma de la medicación, o los de Hipócrates sobre la tendencia de algunos pacientes a abandonar los tratamientos (1). Para la Organización Mundial de la Salud (OMS), la adherencia terapéutica se define como El grado en que el comportamiento de una persona - tomar medicamento, seguir un régimen alimentario y ejecutar cambios del modo de vida - se corresponde con las recomendaciones acordadas de un prestador de asistencia sanitaria ${ }^{(2)}$.

Los efectos de la no adherencia terapéutica repercuten en los aspectos clínicos, psicosociales y económicos de las personas que requieren tratamientos prolongados. En el aspecto clínico, se manifiesta en las recaídas intensas, aparición de complicaciones, efectos adversos y el riesgo de dependencias a determinados fármacos ${ }^{(3)}$. La no adherencia reduce los beneficios del tratamiento, sesga la evaluación clínica de la eficiencia del tratamiento, conlleva a la prescripción de dosis de medicamentos más altas y, en algunos casos, la prescripción de medicamentos que no son necesarios ${ }^{(4)}$. En el aspecto psicosocial, se presenta un estrés crónico ante una enfermedad sintomática que se mantiene en el tiempo ${ }^{(3,5)}$. El aspecto económico, se manifiesta en los gastos por concepto de ausentismo laboral a causa de la enfermedad, con la consecuente repercusión en la productividad de su puesto de trabajo y en pérdidas sensibles en la economía familiar ${ }^{(3)}$.

Por otro lado, los efectos negativos de la no adherencia terapéutica sobre el sistema de salud, se manifiesta en el incremento del gasto en salud, debido a las consultas de salud previamente planificadas que no se ejecutan por la inasistencia de los usuarios, aumento de las intervenciones, hospitalizaciones y tratamientos de alta complejidad a consecuencia de complicaciones generadas por esta conducta ${ }^{(3)}$. La OMS, señala que la interrupción o abandono de una terapia encarece al menos en un $20 \%$ los costos de la salud pública ${ }^{(2)}$. La no adherencia terapéutica está presente en todas las áreas de la práctica clínica. De hecho, han sido ampliamente reportados los bajos niveles de adherencia en una variedad de situaciones, condiciones y poblaciones ${ }^{(5-11)}$.

Este problema se exacerba en pacientes de bajos ingresos económicos, en quienes se han encontrado frecuencias de incumplimiento terapéutico de hasta un $65 \%$ (4). Igualmente, en personas que requieren de tratamientos a largo plazo, se ha estimado que un $80 \%$ no cumple con las indicaciones otorgadas por el equipo de salud (4). La gran magnitud de la no adherencia terapéutica, la hacen una problemática vigente en los sistemas de salud, y que constituye uno de los principales desafíos que se enfrentan los diversos agentes de salud al momento de realizar y planificar intervenciones y estrategias en los usuarios con enfermedades cardiovasculares. La OMS, señala que la medición y la evaluación de la adherencia terapéutica es una necesidad continua, que permite la planificación de tratamientos efectivos, eficientes y de calidad (2). Al respecto, este estudio tuvo como objetivo analizar la adherencia terapéutica en usuarios del programa de salud cardiovascular de atención primaria, en la comuna de San Pedro de la Paz en la Región del Bío Bío en Chile.

\section{MATERIALES Y MÉTODOS}

\section{DISEÑO Y POBLACIÓN DE ESTUDIO}

Se diseñó un estudio transversal y correlacional, cuya unidad de análisis fueron los pacientes de 18 a 60 años de edad pertenecientes al programa de salud cardiovascular de los centros de salud familiar de la comuna de San Pedro de la Paz en la región del Bío Bío en Chile. Los criterios de inclusión fueron: indicación de terapia farmacológica, que tuvieran más de dos años de ingreso al programa de salud cardiovascular, que no tuvieran antecedentes de otras enfermedades crónicas, tal como hipo o hipertiroidismo, cáncer, artrosis y epilepsia, y que no presentaran algún deterioro cognitivo, sensorial, estado grave de salud y/o problemas de salud mental que impidieran responder las preguntas del cuestionario.

Del universo de 2999 pacientes, 804 cumplieron con los criterios de inclusión de las cuales se obtuvo una muestra estratificada al azar de 257 personas. La recolección de datos se realizó entre los meses de noviembre del 2013 y enero de 2014.

\section{VARIABLES E INSTRUMENTOS UTILIZADOS}

Se revisaron las fichas clínicas para conocer los datos relacionados con la enfermedad cardiovascular: diagnóstico médico, índice de masa corporal, circunferencia de la cintura, riesgo cardiovascular, años de hipertensión, años de diabetes, años de dislipidemia y años en el programa de salud cardiovascular. Se aplicaron dos instrumentos en el domicilio del usuario: 
Un cuestionario que permitió recolectar algunos datos sociodemográficos y de su estilo de vida, tal como, edad, sexo, años de educación formal, práctica de ejercicio físico y consumo de tabaco; y el Health Behavior Scale o escala de conductas en salud (ECS), diseñado por Miller et al., con el propósito de medir la adherencia del paciente al régimen terapéutico en base al modelo de creencias en salud. Es una escala tipo diferencial semántica con cinco opciones de respuesta que van desde "en desacuerdo" a "de acuerdo". Cuenta con cinco subescalas relacionadas con el seguimiento de la dieta, limitación en consumo de tabaco, seguir actividades prescritas, tomar medicamentos y el manejo del estrés, propuestos en cuatro enunciados que indagan el cumplimiento en el contexto del hogar, el trabajo, las actividades recreativas y sociales. El puntaje de la escala fluctúa entre 20 a 100. A medida que aumenta el puntaje, mayor es la adherencia terapéutica ${ }^{(12)}$. Está compuesta por dos partes: la parte $A$, que va dirigida al paciente, y la parte $B$, que se aplica en forma independiente a un miembro de la familia que sea significativo para el paciente. El propósito de la parte B es validar las respuestas del paciente al régimen terapéutico. Ambas partes contienen las mismas subescalas, preguntas y opciones de respuesta. Solo cambia la forma a quien va dirigido el enunciado ${ }^{(12)}$. En Chile, esta escala traducida al español como escala de conductas en salud de Miller fue validada en pacientes con antecedentes de infarto e hipertensos ${ }^{(12-15)}$.

\section{PRUEBA PILOTO}

Se realizó una prueba preliminar de ajuste $(n=16)$, que determinó que las respuestas de la escala original se modificaran desde nunca (1 punto) hasta siempre (5 puntos). Posteriormente, se aplicó la parte A de la ECS a pacientes con enfermedades cardiovasculares $(n=208)$ y la parte $B$ a un familiar significativo $(n=208)$. Los coeficientes de correlación de Pearson entre la parte $A$ y la $B$, indicaron relaciones significativas entre las respuestas de los participantes y las respuestas del familiar en todas las subescalas: dieta $(p=0,01)$, limitar el consumo de tabaco $(p=0,01)$, seguir las actividades prescritas $(p=0,01)$, toma de medicamentos $(p=0,01)$, y manejo del estrés $(p=0,01)$. Lo anterior señala que las respuestas de la parte $A$ y de la B son similares en materia de adherencia. Por tanto, se decidió aplicar solamente la parte A en este estudio. Se obtuvieron los siguientes valores de alfa de Cronbach para las subescalas: 0,93 para "seguimiento de la dieta"; 0,98 para "limitación en el consumo de tabaco"; 0,74 para "seguimiento de las actividades prescritas"; 0,88 para el "seguimiento del tratamiento con medicamentos"; y 0,77 para "seguimiento en el manejo del estrés.

\section{ANÁLISIS ESTADÍSTICO}

Se utilizó análisis descriptivo univariante, medidas de tendencia central, de dispersión; y análisis bivariado, correlación de Pearson y ANOVA, apoyado por el software SPSS 15.

\section{CONSIDERACIONES ÉTICAS}

Los participantes firmaron un consentimiento informado. La investigación fue evaluada y autorizada por el Comité de Ética de la Facultad de Medicina de la Universidad de Concepción.

\section{RESULTADOS}

Del total de participantes, $157(61,1 \%)$ fueron mujeres. La media de la edad fue de 50,47 años y la media de los años de educación formal de los participantes fue de 8,58 años (mínima: 0; máxima: 17), solo 40 (15,6\%) manifestaron practicar ejercicio físico al menos tres veces a la semana entre 30 a 45 minutos. Respecto al diagnóstico médico, $195(75,8 \%)$ presentaron hipertensión arterial (HTA), $124(48,2 \%)$ tenían diabetes mellitus tipo 2 (DM2), y 166 (64,5\%) presentaron dislipidemia. La Tabla 1 muestra las particularidades relacionadas con las características de la enfermedad.

Respecto a los enunciados de la ECS, se encontró que del total de la muestra: 57 (22,2\%) fumaban; 141 (54,9\%) tenían trabajo remunerado; $180(70 \%)$ participaban en actividades recreativas, mientras que $93(36,2 \%)$ participaban en actividades sociales. Solo $13(5,1 \%)$ cumplieron con todas las condiciones enunciadas en la escala, es decir, tenían un trabajo remunerado, fumaban, participaban en actividades recreativas o deportivas y sociales. Esta situación determinó que los puntajes mínimos y máximos esperados fueran distintos en los grupos de la muestra, por ejemplo, entre los que trabajan y los que no trabajan; los que fuman y no fuman, entre otros. Para poder efectuar las comparaciones entre

Tabla 1. Medidas de tendencia central y dispersión de las características de la condición de salud de la muestra $(n=257)$

\begin{tabular}{lccc}
\hline \multicolumn{1}{c}{ Características } & Media & Mínima & Máxima \\
\hline Indice de masa corporal & 31,7 & 19,4 & 54,4 \\
Circunferencia de la cintura & 102,2 & 70 & 169 \\
Riesgo cardiovascular & 9,3 & 1 & 20 \\
Años en el programa de salud & 6,6 & 2 & 28 \\
cardiovascular & 8,7 & 1 & 30 \\
Años de hipertensión arterial & 7,5 & 1 & 28 \\
Años de diabetes mellitus tipo 2 & 4,8 & 1 & 28 \\
Años de dislipidemia & & & \\
\hline
\end{tabular}


Tabla 2. Medidas de tendencia central y dispersión de la escala de conductas en salud de Miller

\begin{tabular}{lccc}
\hline \multicolumn{1}{c}{ Escala de conductas en salud } & $\mathbf{n}$ & Media & D. E \\
\hline Seguimiento de la dieta & 257 & 3,2 & 1,1 \\
Limitación consumo de tabaco & 57 & 3,1 & 1,0 \\
Seguimiento actividades prescritas & 257 & 3,0 & 1,2 \\
Seguimiento toma de medicamentos & 257 & 3,1 & 1,5 \\
Seguimiento en el manejo del estrés & 257 & 4,1 & 1,0 \\
Escala global & 257 & 3,4 & 1,4 \\
\hline
\end{tabular}

los participantes, se decidió estandarizar los puntajes obtenidos por cada usuario en una escala de 1 a 5 .

Respecto a los puntajes medios estandarizados de la ECS, la subescala seguimiento en el manejo del estrés tuvo la media más alta, lo que indica una mayor adherencia en los participantes. El resto de las subescalas y la escala global, presentaron puntajes medios similares (Tabla 2).

En relación a la distribución de la muestra según los puntajes estandarizados obtenidos en la ECS (Tabla $3)$, la escala global refleja la no adherencia de los participantes, ya que solo $4(1,5 \%)$ personas obtuvieron el puntaje máximo, es decir, que siempre siguen las indicaciones entregadas por el equipo de salud. Más de la mitad de la muestra $(73,5 \%)$ indicó que nunca, casi nunca o a veces siguen las indicaciones, lo que se considera una adherencia baja, dada la importancia que tiene el cumplimiento continuo o permanente del tratamiento en las enfermedades crónicas.

Se encontraron correlaciones positivas entre la edad del participante con el seguimiento de las actividades prescritas $(p=0,034)$; el seguimiento en el manejo del estrés $(p=0,007)$; y la escala global $(p=0,026)$. También se encontró una correlación positiva entre los años de estudio y el seguimiento en la toma de medicamentos $(p=0,036)$.

Respecto a la comparación de la adherencia terapéutica entre grupos (Tabla 4), las mujeres fueron más adherentes en la toma de medicamentos, los fumadores fueron menos adherentes a la toma de medicamentos, la realización de un trabajo remunerado se relacionó con menor adherencia al seguimiento de la dieta y en la escala global, la participación en actividades recreativas se asoció al manejo del estrés, asimismo la participación en actividades sociales tuvo una relación significativa con el seguimiento en la toma de medicamentos, manejo del estrés y la escala global. No se encontraron asociaciones entre en número de enfermedades que tiene el participante y la conducta de adherencia (ANOVA: $p=0,510$ ).

\section{DISCUSIÓN}

La baja adherencia terapéutica encontrada es similar a otros estudios realizados en grupos de personas con enfermedades cardiovasculares ${ }^{(5,6,8,9,11)}$ y coincide con los bajos niveles de control de las enfermedades cardiovasculares que tiene Chile ${ }^{(18)}$, es decir, la baja prevalencia de pacientes que logran alcanzar la metas terapéuticas propuestas para la HTA, DM2 y dislipidemias.

La alta frecuencia de participantes que no siempre siguen la dieta indicada es concordante con el alto índice de masa corporal y circunferencia de cintura encontrada en la muestra. Lo que da cuenta de la mal nutrición por exceso de los pacientes cardiovasculares, similar a lo reportado en la población general a nivel país ${ }^{(16)}$.

Se encontró un bajo porcentaje de fumadores en la muestra $(22,2 \%)$ en comparación a la presentada en la población general en el año $2010(40,6 \%)^{(16)}$. A pesar de esta baja prevalencia de fumadores, estos presentaron una baja frecuencia en el seguimiento de la limitación del consumo de tabaco, solo cinco fumadores indicaron que siempre limitaban o disminuían el consumo de tabaco en todos los ámbitos propuestos por la escala.

Respecto al seguimiento de las actividades prescritas, las cuales se enfocan principalmente en la realización de actividad y/o ejercicio físico, fue baja, ya que más de la mitad de los participantes refirió que nunca, casi nunca o a veces tenían esta conducta. En estos

Tabla 3. Distribución de la muestra según puntajes estandarizados de la escala de conductas en salud de Miller

\begin{tabular}{lccccc}
\hline \multicolumn{1}{c}{ Escala de conductas en salud } & Nunca & Casi nunca & A veces & Casi siempre & Siempre \\
\hline Seguimiento de la dieta & $22(8,6 \%)$ & $58(22,5 \%)$ & $93(36,2 \%)$ & $64(24,9 \%)$ & $20(7,8 \%)$ \\
Limitación en consumo de tabaco & $5(8,8 \%)$ & $15(26,3 \%)$ & $21(36,8 \%)$ & $14(24,6 \%)$ & $2(3,5 \%)$ \\
Seguimiento de actividades prescritas & $36(14 \%)$ & $66(25,7 \%)$ & $75(29,2 \%)$ & $51(19,8 \%)$ & $29(11,3 \%)$ \\
Seguimiento de toma de medicamentos & $47(18,3 \%)$ & $68(26,4 \%)$ & $38(14,9 \%)$ & $32(12,4 \%)$ & $72(28 \%)$ \\
Seguimiento en el manejo del estrés & $9(3,5 \%)$ & $22(8,6 \%)$ & $43(16,7 \%)$ & $88(34,3 \%)$ & $95(36,9 \%)$ \\
Escala global & $8(3,1 \%)$ & $77(29,9 \%)$ & $104(40,5 \%)$ & $64(24,9 \%)$ & $4(1,6 \%)$ \\
\hline
\end{tabular}


Tabla 4. Escala de conductas en salud de Miller y variables sociodemográficas y de la enfermedad crónica

\begin{tabular}{|c|c|c|c|c|c|c|}
\hline Variable & $\begin{array}{l}\text { Seguimiento } \\
\text { de la dieta }\end{array}$ & $\begin{array}{c}\text { Limitación en } \\
\text { consumo de tabaco }\end{array}$ & $\begin{array}{l}\text { Seguimiento de } \\
\text { actividades prescritas }\end{array}$ & $\begin{array}{l}\text { Seguimiento de toma } \\
\text { de medicamentos }\end{array}$ & $\begin{array}{l}\text { Seguimiento en el } \\
\text { manejo del estrés }\end{array}$ & $\begin{array}{l}\text { Escala } \\
\text { global }\end{array}$ \\
\hline \multicolumn{7}{|l|}{ Sexo } \\
\hline Hombre & 3,1 & 3,2 & 3,1 & $2,9^{*}$ & 4,2 & 3,3 \\
\hline Mujer & 3,2 & 3,1 & 3,0 & 3,2 & 4,0 & 3,3 \\
\hline \multicolumn{7}{|c|}{ Hábito tabáquico } \\
\hline No & 3,2 & - & 3,0 & $3,2^{*}$ & 4,1 & 3,3 \\
\hline Sí & 3,1 & - & 3,2 & 2,7 & 4,2 & 3,3 \\
\hline \multicolumn{7}{|l|}{ Trabajo } \\
\hline No & $3,3^{*}$ & 3,2 & 3,1 & 3,3 & 4,0 & $3,4^{*}$ \\
\hline Sí & 3,0 & 3,0 & 2,9 & 2,9 & 4,1 & 3,2 \\
\hline \multicolumn{7}{|c|}{ Participación en actividades recreativas } \\
\hline No & 3,2 & 3,2 & 3,0 & 3,1 & $3,8^{*}$ & 3,3 \\
\hline Sí & 3,1 & 3,1 & 3,0 & 3,1 & 4,2 & 3,3 \\
\hline \multicolumn{7}{|c|}{ Participación en actividades sociales } \\
\hline No & 3,1 & 3,0 & 2,9 & $2,9^{*}$ & $3,9^{*}$ & $3,2^{*}$ \\
\hline Sí & 3,3 & 3,4 & 3,1 & 3,4 & 4,3 & 3,5 \\
\hline
\end{tabular}

${ }^{*} p<0,05$ para la $t$ de Student

resultados el $84,4 \%$ de los participantes manifestó ser sedentario, este porcentaje es similar a la prevalencia de sedentarismo a nivel nacional ${ }^{(16)}$. Esta situación responde a la influencia de diversos factores sociales y culturales que dificultan la práctica de ejercicio físico.

Llama la atención que solo el $28,01 \%$ de la muestra declaró seguir siempre las indicaciones de la toma de medicamentos. Este resultado fue mucho menor a lo encontrado en otros estudios realizados en pacientes diabéticos ${ }^{(17,18)} \mathrm{e}$ hipertensos ${ }^{(8)}$. Se esperaba que este porcentaje fuese más alto debido a que en Chile el acceso a los medicamentos para las enfermedades cardiovasculares está garantizado por ley a través de los planes de las Garantías Explicitas en Salud (GES). Lo anterior se podría explicar por otros factores que podrían estar influyendo negativamente en la toma de los medicamentos, tales como la polifarmacia, dosificación del medicamento, el olvido de tomarse el medicamento, percepción de fracaso en tratamientos anteriores, entre otros, los cuales son interesantes de seguir indagando a través de investigaciones.

Con relación al seguimiento del manejo del estrés, este presentó el porcentaje más alto de adherencia en la muestra. Sin embargo, se esperaba que la adherencia en el manejo del estrés fuese más baja, considerando lo complejo que significa vivir con una enfermedad crónica. Lo anterior podría explicarse por una baja percepción de estrés en los pacientes, concordante con la población general. De acuerdo a la Encuesta Nacional de Salud del 2010, en Chile la prevalencia de percepción de estrés permanente en el último año fue de un $8,8 \%$, y de percepción de estrés financiero fue de un $18,1 \%{ }^{(16)}$.
Las mujeres fueron más adherentes en la toma de medicamentos. Este aspecto se podría explicar por factores culturales, en los cuales el rol de la mujer se enmarca como cuidadora en el contexto familiar, lo que podría ser significativo al momento de tomar de decisiones en el seguimiento del tratamiento farmacológico.

Los años de estudio se asociaron al seguimiento de la toma de medicamentos, a medida que aumenta la escolaridad, aumenta el puntaje de adherencia en la toma de medicamentos. Esto puede explicarse por un lado, porque el nivel de escolaridad puede interferir en la comprensión y aceptación de la enfermedad y, por tanto, en llevar a cabo el tratamiento farmacológico. La muestra presentó un bajo nivel de escolaridad, lo cual podría constituir una barrera en la toma de decisiones relacionadas con su salud o para movilizar recursos y redes comunitarias ${ }^{(11)}$. Este factor se considera relevante para el equipo de salud al momento de educar y empoderar a las personas respecto a las enfermedades crónicas y los tratamientos de largo plazo.

Las personas que trabajaban presentaron puntajes más bajos en la adherencia a la dieta y la escala global, coincidente con otros estudios ${ }^{(19)}$. Esto podría estar relacionado, con la presencia de obligaciones que dificultan la falta de cuidado de su enfermedad cardiovascular o con las largas jornadas laborales que pueden constituir una barrera al momento de llevar un régimen alimentario. La situación anterior da cuenta de la necesidad de incorporar estrategias flexibles en los centros de salud familiar que garanticen la continuidad 
de los cuidados en las personas que trabajan desde una perspectiva integradora.

La participación en actividades recreativas y sociales favoreció la adherencia al manejo del estrés, esto se puede explicar por los beneficios físicos, sociales, intelectuales, psicológicos y espirituales que entrega la práctica de actividades que proporcionan placer durante el tiempo libre ${ }^{(20-22)}$

De acuerdo a la OMS no existe un patrón de oro para medir este comportamiento, existen diversas formas de cuantificar este fenómeno, cada una con sus limitaciones y ventajas ${ }^{(2)}$. Una limitación de la ECS de Miller, es que los enunciados exigen ciertas condiciones, tales como fumar, trabajar, participar en actividades recreativas o sociales, y no todas las personas de la muestra presentaron esas condiciones, por tanto, un grupo importante quedó sin contestar todos los ítems de la escala. La estandarización de los puntajes permitió efectuar comparaciones y analizar los resultados de la muestra. Esta situación hace que la ECS sea difícil de aplicar en el cotidiano del trabajo en los equipos de salud de atención primaria, considerando el poco tiempo con el que cuentan para la atención de los pacientes con enfermedades cardiovasculares y la escasez de profesionales en este nivel de atención. A pesar de lo anterior, una de las ventajas de la ECS, es que permite indagar sobre las conductas de adherencia en todos los escenarios en que se desenvuelve la vida de las personas, lo que favorece la detección en forma adecuada la no adherencia terapéutica ${ }^{(6)}$. Una limitación de esta investigación es que sus resultados no pueden generalizarse y solo son aplicables a la población en estudio.

Se concluye que en la adherencia al tratamiento de las enfermedades cardiovasculares pueden influir diversos factores sociales que pueden constituir una barrera al momento de cumplir con el tratamiento, tales como el nivel socioeconómico y educacional, el apoyo social, entornos que permitan la práctica de ejercicio, ambientes libres de humo de tabaco, políticas de salud que garanticen el acceso al tratamiento en forma oportuna y de calidad, entre otros. Lo anterior indica la importancia de desarrollar estrategias en los programas de salud cardiovascular que favorezcan la adherencia terapéutica desde la perspectiva de equidad y de los determinantes sociales de la salud.

Contribuciones de los autores: LVR participó en la concepción y diseño del artículo; recolección de resultados, análisis e interpretación de datos; redacción y revisión crítica del artículo; aprobación de su versión final; aporte de pacientes o material de estudio y obtención de financiamiento. SMP y $O A B$ participaron en el análisis e interpretación de datos; revisión crítica y aprobación de la versión final del artículo; y asesoría técnica o administrativa.

Fuentes de financiamiento: estudio financiado por CONICYT a través de "beca gastos operacionales para tesis doctoral", y por el "Convenio de desempeño vida saludable CD UCO 1201, Universidad de Concepción".

Conflictos de interés: los autores declaran no tener conflicto de intereses.

\section{REFERENCIAS BIBLIOGRÁFICAS}

1. Orueta R, Toledano P, GomézCalcerrada RM. Actualización en medicina de familia: cumplimiento terapéutico. Semergen. 2008;34(5):235-43.

2. Organización Mundial de la Salud. Adherencia a los tratamientos a largo plazo. Pruebas para la acción. Ginebra: OMS; 2004.

3. Libertad M. Repercusiones para la salud pública de la adherencia terapéutica deficiente. Rev Cubana Salud Publica. 2006; 32(3).

4. D'Anello S. Instrumento para medir variables psicosociales asociadas al cumplimiento del tratamiento médico. MedULA, Revista de Facultad de Medicina. 2006;15(1):4-11.

5. La Rosa Y, Libertad M, Bayarre H.
Adherencia terapéutica y factores psicosociales en pacientes hipertensos. Rev Cubana Med Gen Integr. 2007;23(1).

6. Mendoza $S$, Muñoz M, Merino JM, Barriga OA. Factores determinantes de cumplimiento terapéutico en adultos mayores hipertensos. Rev Med Chile. 2006;134(1):65-71.

7. Zugelj U, Zupancic M, Komidar L, Kenda R, Varda NM, Gregoric A. Self-reported adherence behavior in adolescent hypertensive patients: the role of illness representations and personality. J Pediatr Psychol. 2010 Oct;35(9):1049-60. doi: 10.1093/ jpepsy/jsq027.

8. Mizuno R, Fujimoto S, Uesugi A, Danno D, Maeda K, Kanno M, et al. Influence of living style and situation on the compliance of taking antihypertensive agents in patients with essential hypertension. Intern Med. 2008;47(19):1655-61.

9. Linck C, Bielemann V, de Sousa A, Lange C. The chronic patient in face of falling ill and the treatment compliance. Acta Paul Enferm. 2008;21(2):317-22.

10. Ahmed N, Abdul Khaliq M, Shah $\mathrm{SH}$, Anwar W. Compliance to antihypertensive drugs, salt restriction, exercise and control of systemic hypertension in hypertensive patients at Abbottabad. J Ayub Med Coll Abbottabad. 2008 Apr-Jun;20(2):66-9.

11. Mendoza-Parra S, Merino JM, Barriga OA. Identificación de factores de predicción del incumplimiento terapéutico en adultos mayores hipertensos de una comunidad del sur 
de Chile. Rev Panam Salud Publica. 2009;25(2):105-12.

12. Miller P, Johnson NL, Garrett MJ, Wickoff R, McMahon M. Health beliefs of and adherence to the medical régimen by patients with ischemic heart disease. Heart Lung. 1982 JulAug;11(4):332-9.

13. Miller P, Wikoff RL, McMahon M, Garrett MJ, Ringel K. Indicators of medical régimen adherence for myocardial infarction patients. Nurs Res. 1985 Sep-Oct;34(5):268-72.

14. Miller P, Wikoff R, Keen O, Norton J. Health belief and regimen adherence of the American Indian diabetic. Am Indian Alsk Native Ment Health Res. 1987;1(1):24-36.

15. Mendoza PS, Figueroa FM. Creencias en salud $y$ adherencia de pacientes hipertensos controlados en centros de salud de la comuna de Concepción, Chile. Cienc Enferm. 1998;41(1):63-76.

16. Ministerio de Salud de Chile. Encuesta nacional de Salud 2009-2010 [Internet].
Santiago de Chile: MINSAL; 2010 [citado 02 de septiembre 2012]. Disponible en: http://epi.minsal.cl/ estudios-y-encuestas-poblacionales/ encuestas-poblacionales/descarga-ens/

17. Aikens JE, Piette JD. Longitudinal association between medication adherence and glycemic control in type 2 diabetes. Diabet Med. 2013 Mar;30(3):338-44. doi: 10.1111/ dme.12046.

18. Pascal IG, Ofoedu JN, Uchenna NP, Nkwa AA, Uchamma GU. Blood Glucose Control and Medication Adherence Among Adult Type 2 Diabetic Nigerians Attending A Primary Care Clinic in Underresourced Environment of Eastern Nigeria. N Am J Med Sci. 2012 Jul;4(7):310-5. doi: 10.4103/19472714.98590 .

19. Corugedo M, Martín L, Bayarre H. Adherencia terapéutica en pacientes con hipertensión arterial del policlínico universitario "Manuel Fajardo" en el municipio Cruces, Cienfuegos 2009. Rev Cubana Med Gen Integr. 2011;27(4):504-12.

20. Salazar C. Recreación. San José: Universidad de Costa Rica; 2007.

21. Quintana A, Merino JM, Merino P, Cea JC. Variables psicosociales asociadas a compensación metabólica de pacientes diabéticos tipo 2. Rev Med Chile. 2008;136:1007-14.

22. Gomes-Villas L, Foss M, Foss de Freitas M, Pace A. Relación entre apoyo social, adhesión al tratamiento y control metabólico de personas con diabetes mellitus. Rev Latino-Am Enfermagem 2012;20(1).

Correspondencia: Lizet Veliz-Rojas Dirección: Universidad Católica del Norte. Facultad de Medicina, Campus Guayacán. Larrondo 1281, Coquimbo, Chile. Teléfono: +56997702788

Correoelectrónico:lizet.veliz@ucn.cl

\section{Consulte la versión electrónica de la Revista Peruana de Medicina Experimental y Salud Pública en} www.pubmed.gov

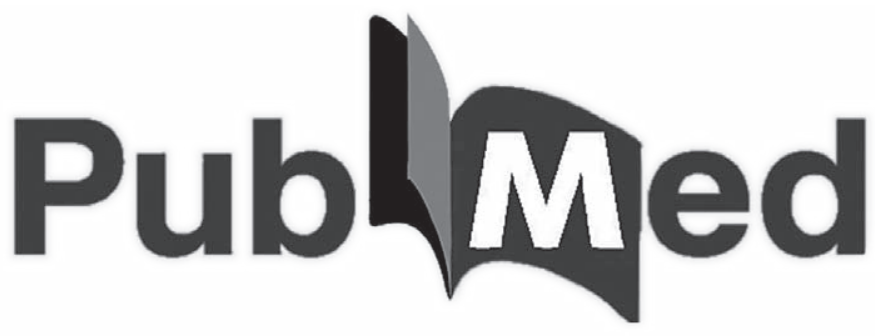

